\title{
Laparoscopic nephroureterectomy with transvesical single-port distal ureter and bladder cuff dissection: points of technique and initial surgical outcomes with five patients
}

\author{
Marek Roslan, Marcin Markuszewski, Jakub Kłącz, Marcin Sieczkowski, Wojciech Połom, Wojciech Piaskowski, \\ Kazimierz Krajka, Marcin Matuszewski \\ Department of Urology, Medical University of Gdansk, Poland
}

Videosurgery Miniinv 2014; 9 (2): 267-272 DOI: $10.5114 /$ wiitm.2013.39518

\begin{abstract}
Although a variety of techniques have been used to manage the distal ureter during laparoscopic radical nephroureterectomy (LNU), a consensus has not yet been established. Recently, some authors have used a single-port transvesical approach to excise the distal ureter and bladder cuff following LNU. The aim of the study was to present our initial experience in "en bloc" dissection of the distal ureter and bladder cuff during LNU, using a transvesical single-port approach (T-LESS) and standard laparoscopic instruments. From April to October 2012, 5 patients aged 45 to 73 years with upper urinary tract urothelial tumors were subjected to LNU/T-LESS. After a standard LNU was performed, a TriPort ${ }^{\oplus}$ device was introduced into the bladder and the pneumovesicum was established. A bladder cuff with a distal ureter was dissected and put in the paravesical tissue. The bladder wall defect was closed with the $V$-loc ${ }^{\circledast}$ 3/O suture. The LNU was then completed in the flank position. All procedures were completed successfully. No significant blood loss or complications were observed. The mean operative time was $250 \mathrm{~min}$ (range: 200-370) for a total procedure and $59 \mathrm{~min}$ (range: 42-80) for the T-LESS stage. The postoperative hospital stay was 5.2 days (range: 4-9). Pathologic examination revealed no positive margin in any of the cases. The LNU/T-LESS approach is an efficient and safe procedure. A well-visualized dissection of the distal ureter, closing the defect of the bladder, the use of standard laparoscopic instruments and a good cosmesis are advantages of the method.
\end{abstract}

Key words: transitional cell carcinoma, upper urinary tract, minimally invasive surgery, laparoendoscopic single-site surgery.

\section{Introduction}

Since Clayman et al. [1] first performed laparoscopic nephroureterectomy (LNU) for upper urinary tract transitional cell carcinoma, a variety of techniques have been used to manage the distal ureter during LNU, but a consensus has not yet been established. To date, excision of the distal ureter and bladder cuff following LNU has been performed by different approaches, namely, open excision (intravesical or extravesical), transurethral access (pluck method or the intussusception technique), or the extravesical method such as the use of staples [2-4]. With the advances in endoscopic equipment, transvesical approaches, either with standard filling with glycine or with carbon dioxide, have become more widely used [5-7]. One of the latest methods is transvesical laparoendoscopic single-site surgery (T-LESS), introduced by Sotelo et al. in 2011 [8].

In this paper we present our preliminary experience in en-bloc dissection of the distal ureter and bladder cuff during LNU, using a transvesical sin- 


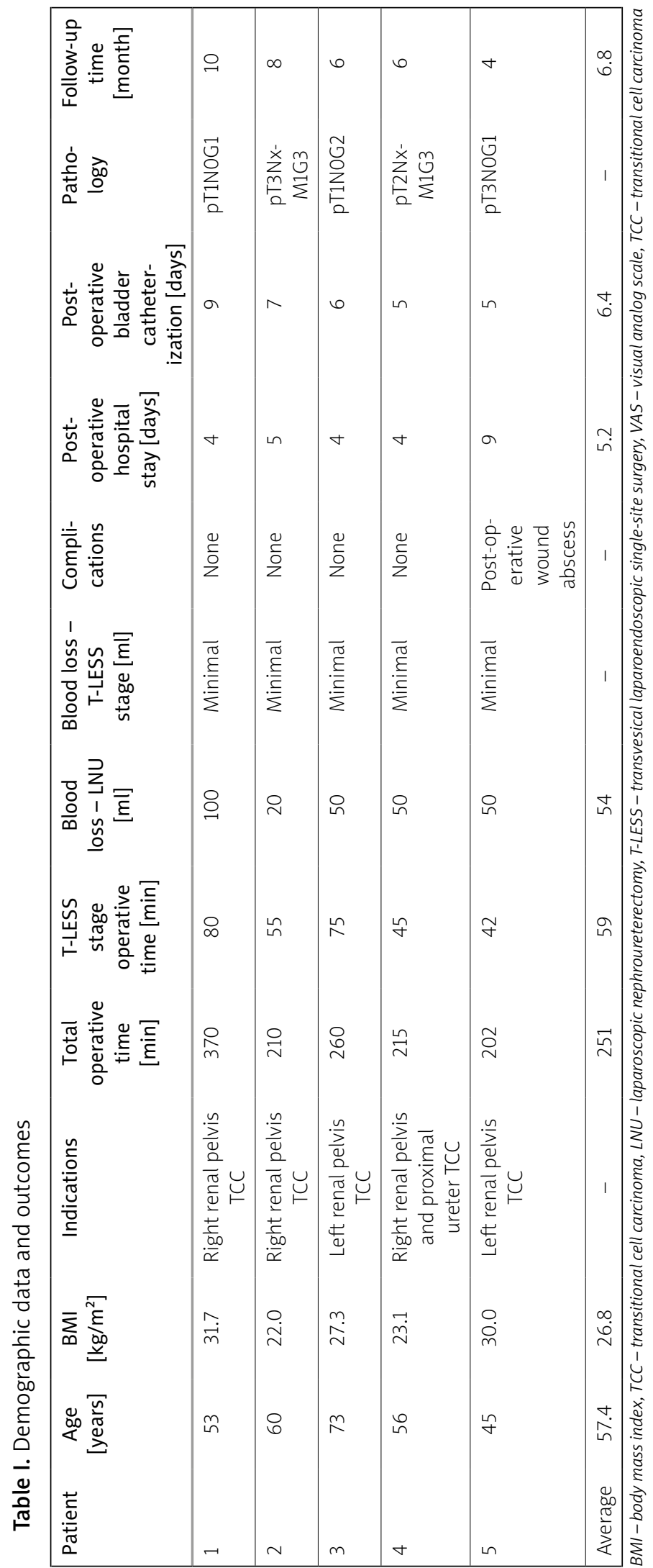

gle-port approach (T-LESS) and standard laparoscopic instruments. We also elaborate on the technique, which has been slightly modified according to the principles described by Sotelo's team.

The aim of the study was to present our experience of distal ureter and bladder cuff excisions, as a stage of laparoscopic nephroureterectomy, using a single port introduced directly into the bladder.

\section{Case reports}

In this case series study we present our initial clinical results with 5 patients who were operated on for upper urinary tract urothelial tumors with enbloc laparoscopic nephroureterectomy and transvesical laparoendoscopic single-site distal ureter and bladder cuff excision (LNU/T-LESS). The patient data are presented in Table I.

Surgery was performed after obtaining informed consent from all patients, and approval by the local ethics committee.

The procedures were performed between April and October 2012. The principles of the technique have been described previously $[6,8]$. Patients were operated on under general anesthesia. They were placed in the flank position and standard four-port LNU was performed. During this step, after early renal vessel ligation, the juxtavesical ureter was occluded with a metal or hem-o-loc clip as distally as possible (Photo 1).

Then, the patients were placed in the lithotomy position. After filling the bladder with $300 \mathrm{ml}$ of sterile $0.9 \%$ saline, under cystoscopic control, a single-port access system (TriPort+ ${ }^{\oplus}$, Olympus, Germany) was inserted into the bladder through a $1.5-\mathrm{cm}$ skin incision, made $2-3 \mathrm{~cm}$ above the pubic symphysis (Photo 2). In 3 obese patients, a 1-cm in length rectus sheath incision was made, and stay sutures were placed in two of them to facilitate introduction of the port. Next, a pneumovesicum with carbon dioxide to a pressure of $14 \mathrm{~mm} \mathrm{Hg}$ was established, and a standard, rigid, $0^{\circ}$ or $30^{\circ}$ optic (Olympus Europa $\mathrm{GmbH}$, Germany) was introduced through the 10-mm channel of the TriPort+. The bladder mucosa was watched carefully and the bladder trigone was exposed. The ipsilateral ureteral orifice was identified and a bladder cuff margin $1.5 \mathrm{~cm}$ in diameter was marked around the orifice using a hook electrode (Photo $3 \mathrm{~A}$ ). The mucosa on the bladder cuff was coagulated to avoid the spread of any neoplastic cells into the abdominal cavity. The cuff and a $4 \mathrm{~cm}$ to 


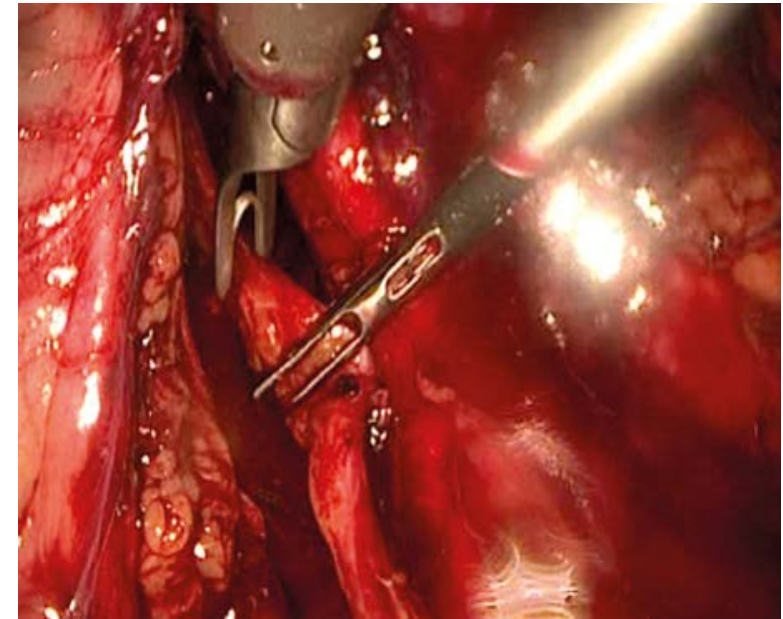

Photo 1. Occlusion of a distal ureter with a metal clip during the LNU step
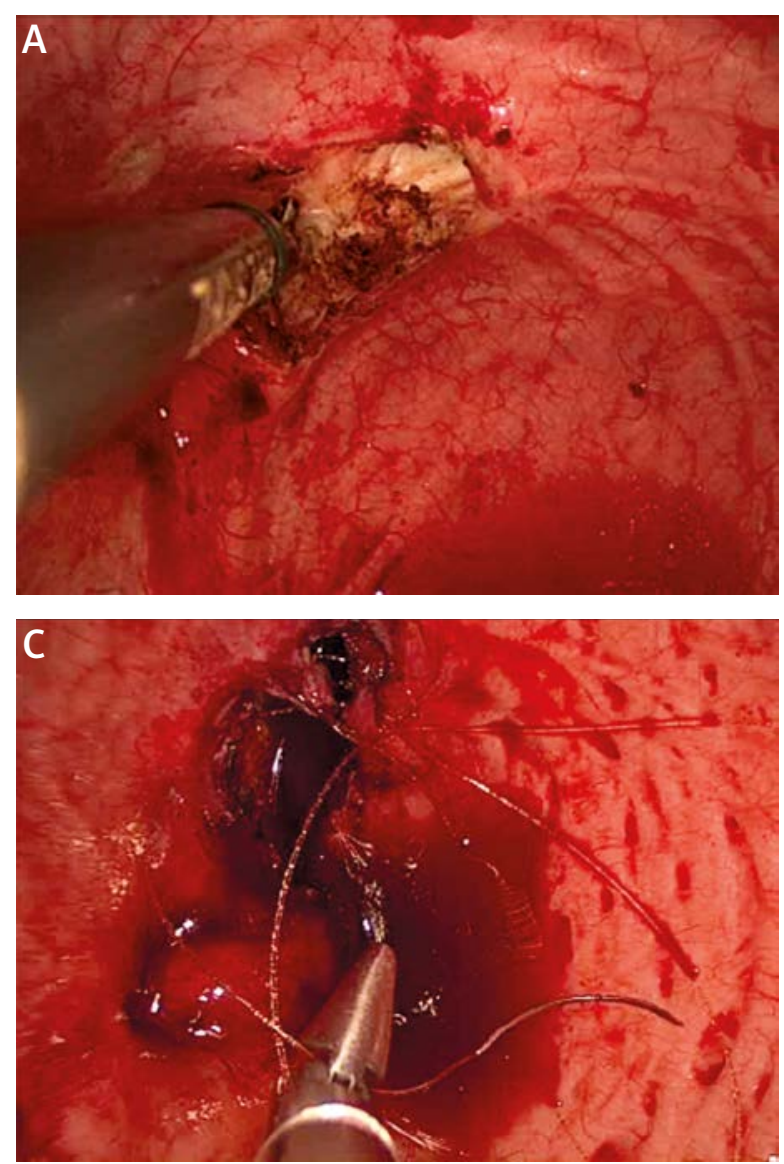

$6 \mathrm{~cm}$ long distal ureter were dissected until the clip was visualized (Photo $3 \mathrm{~B}$ ).

The bladder cuff and the ureter were then placed in the paravesical adipose tissue, and the bladder fundus defect was closed with an absorbable 3/0

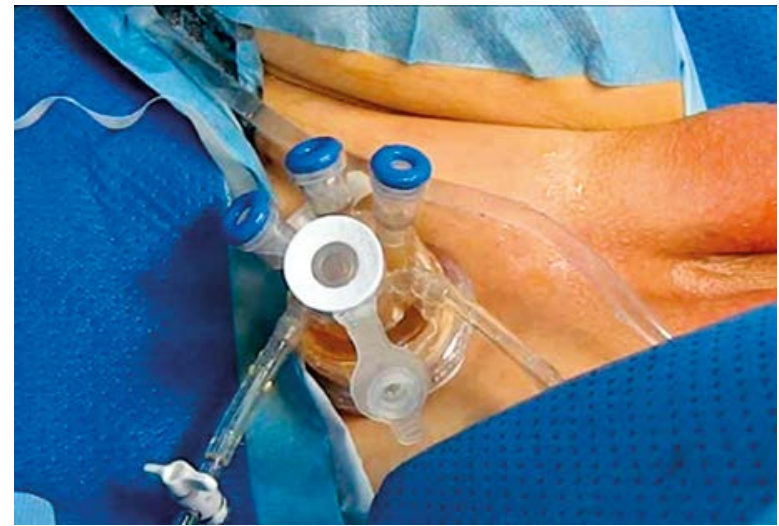

Photo 2. Percutaneous insertion of the TriPort+ into the bladder

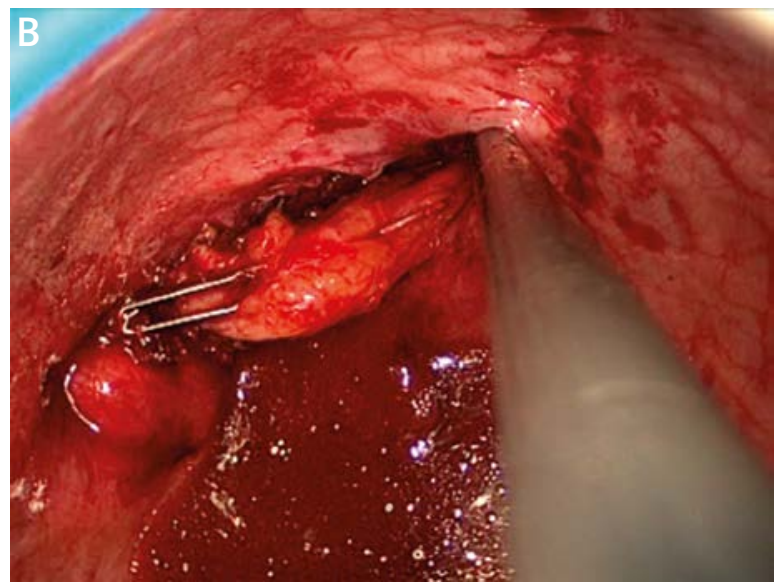

Photo 3. A - Circuitous dissection of the ureteral orifice and bladder cuff with a hook electrode. B - The distal ureter is mobilized until the clip is visualized. C - Closure of the bladder fundus defect with an absorbable 3/0 barbed running suture

barbed running suture (The V-Loc ${ }^{\text {TM }} 90$ Absorbable Wound Closure Device, Covidien, USA) (Photo $3 \mathrm{C}$ ). Finally, an 18 F Foley catheter was introduced into the bladder, the pneumovesicum was evacuated and the single port was removed. The skin incision was 
closed with two stitches. If the rectus sheath was incised previously, it was sutured with one stitch. Then the patient was rotated to the flank position for the completion of LNU. The specimen was extracted in its entirety in a bag through a lower abdominal muscle-splitting incision. An intraperitoneal drainage tube was left for 2-3 days.

In all patients, only standard rigid instruments were used. In the T-LESS stage, when needed, the transurethral access was used for insertion of a suction tube. Moreover, a standard laparoscopic grasper was inserted through the urethra to take the traction of the bladder cuff when needed.

Patients were treated postoperatively with courses of antibiotics, based on the results of urine culture when available. The Foley catheter was removed on the $5^{\text {th }}$ to $9^{\text {th }}$ postoperative days. No imaging was deemed necessary to confirm bladder integrity before catheter removal. In fact, this approach varies in part from the principles described by Sotelo et al. [8]. Moreover, after removal of the TriPort+ the opening in the bladder dome was not sutured. Leaving a Foley catheter for 5-9 days appeared sufficient to obtain proper bladder healing.

The primary goal of this study was to validate the efficacy and safety of the method. The perioperative parameters - operative time, hospital stay, blood loss, catheterization time, and complications - were collected. The visual analog scale (VAS) was used to evaluate postoperative pain. The evaluation of pain was achieved with the documentation of the patient's perceived pain on an analogue pain scale that ranged from 1 to 10 . The follow-up assessments, including history, ultrasonography, biochemistry and urinalysis, were obtained at 6 weeks, and every 3 months following the operation. We arranged for 3-monthly cystoscopy and a computed tomography evaluation after 6 to 12 months.

All patients were managed adequately in the manner presented above. Baseline characteristics and perioperative data of patients are presented in Table I. All procedures were completed successfully. No significant blood loss or complications were observed, except that patient 5 developed a subcutaneous abscess located in the abdominal wall incision, made intraoperatively to remove the specimen. The patient was treated successfully with the narrow opening of the wound, establishing drainage and treating with a prolonged course of antibiotics.
The mean operative time was 251 min (range: 202-370) for the total procedure and 59 min (range: 42-80) for the T-LESS stage. The postoperative hospital stay was 5.2 days (range: 4-9). No adverse events connected with the method were observed during the mean follow-up time of 6.8 months (range: 4-10). Pathologic examination revealed no positive margin in any of the cases.

\section{Discussion}

The primary issue in the surgical treatment of an upper tract carcinoma is the total removal of the kidney, ureter and the bladder cuff that surrounds the ureteral orifice. This principle should be obeyed to prevent local recurrences in intramural ureters, the rate of which can be as high as $16-58 \%[9,10]$.

For complete removal of the upper urinary tract with bladder cuff, two incisions (lumbar and pararectal) were traditionally necessary to provide an adequate operative field [11]. In the era of laparoscopic surgery, LNU has become the standard procedure in the surgical management of patients with upper tract urothelial carcinoma. Laparoscopic nephroureterectomy and the open approach are comparable in terms of perioperative parameters and oncologic efficacy $[12,13]$. Although LNU is more time consuming, it provides less blood loss and less postoperative pain than the open procedure. The other advantages of the technique are quicker oral intake, shorter hospitalization and a more rapid recovery [11].

In principle, the LNU procedures duplicate the open techniques. However, to date, the optimal technique for distal ureter and bladder cuff excision during LNU has not been determined. The laparoscopic extravesical approach was among the first attempted. It was assisted primarily by the use of a stapler (EndoGIA; Autosuture) or Ligasure (Valleylab; Tycohealthcare) [13-15]. The reason this technique was performed is most likely because the laparoscopic extravesical approach is technically less demanding, similar to open surgery. Nevertheless, the use of the stapler may result in the long-term risk of stone formation, and the use of a ligature may result in accidental damage to the opposite ureteral orifice $[16,17]$.

Another group of techniques exploring the transvesical approach was also introduced to follow the steps performed during the open transvesical exci- 
sion technique. For example, Gill et al. described an approach that involves the use of two 2- $\mathrm{mm}$ transvesical suprapubic trocars, a ureteral stent in the ipsilateral ureter and a Collin's knife to excise the ureteral orifice [5]. Similar techniques were reported by Ahlawat and Gautam, and Zou et al.; but they used only one transvesical suprapubic $5-\mathrm{mm}$ or 10 $\mathrm{mm}$ port $[18,19]$. The primary disadvantage of the previously described transvesical techniques is that the bladder wall defect created after the distal ureter and the bladder cuff excision was not closed, resulting in a risk of postoperative urine extravasation [5, 20]. Cheng et al. were the first to duplicate the traditional open transvesical approach for an en-bloc LNU, with pure transvesical laparoscopic distal ureter and bladder cuff excision. They placed three PediPort trocars in the bladder, established a pneumovesicum, and after excision of the orifice with a bladder cuff, they closed the bladder wall defect with a suture, but they did not close the trocar sites [6].

At present, the minimal invasiveness of the direct transvesical suprapubic access has evolved considerably, and has been applied to various indications in bladder surgery. The most interesting techniques seem to be those using transvesical single access systems and establishing a pneumovesicum. To date, simple prostatectomies, diverticulectomies, foreign body removals and ureteral reimplantation have been performed using transvesical laparoendoscopic single-site surgery (T-LESS) [21-24]. The T-LESS approach was combined with LNU first by Sotelo et al. [8]. These authors performed the T-LESS bladder cuff and distal ureter excision following single-port LNU. In all cases they established pneumovesicum, and, after performing distal ureterectomy, they closed the bladder wall defect using intracorporeal suturing. They used the EndoEye 5-mm optics and either standard or articulating laparoscopic instruments. They also closed the anterior cystotomy, made for the placement of the single-port trocar.

In our group of patients we generally followed the principles presented by Sotelo et al. The modification to this procedure is that we used the transurethral access for introducing the laparoscopic grasper or the suction device. This maneuver allowed us to make traction of the bladder cuff and facilitated the dissection of the distal ureter. The grasper inserted through the urethra was also helpful for tightening a suture when the bladder wall defect was fixed. Moreover, the opening in the bladder dome was not closed after the TriPort+ was removed. The single port was introduced bluntly through the detrusor muscle, drawing aside the muscle fibers, which were sealed up after the TriPort+ was removed. The skin incision and the rectus fascia, if it had been cut previously, were sutured. The 4-8 day healing of the bladder, secured by the $18 \mathrm{~F}$ Foley catheter, was sufficient to achieve proper bladder integrity. Urine extravasation after the removal of the catheter was observed in no cases.

In the T-LESS step, the use of cystoscopy is essential to guide the insertion of the port. Moreover, a thorough inspection of the bladder mucosa before the introduction of the TriPort is crucial to avoid the risk of missing either small urothelial carcinomas or, rarely, other tumors [25].

Our early results are generally in accordance with those presented in other series using the pneumovesicum approach $[6,8,19]$. The only complication was an abdominal wall abscess that developed in the wound created for removal of the specimen. In the past, we have observed such abscesses after some laparoscopic operations, especially nephrectomies, and we do not link this complication with the T-LESS procedure.

The main limitations of this work are the small number of patients and the relatively short follow-up time. The drawbacks of the method we present are the necessity for patient repositioning, the relatively small operative space and the clashing instruments during the T-LESS step. Nevertheless, our results confirm the usefulness of the T-LESS approach as a valuable alternative to other techniques applied for distal ureter and bladder cuff excision.

The advantages of this technique are excellent visualization inside the bladder, allowing the identification of all the important structures and the protection of the contralateral orifice, and the use of standard laparoscopic instruments. Furthermore, the watertight closure of the defect in the bladder fundus minimizes the risk of urine extravasation. From an oncological point of view, the other benefit is that the establishment of the pneumovesicum instead of the use of glycine may limit the risk of cancer-cell spillage [6].

The authors would like to emphasize that, to our best knowledge, this is the first case series that confirms the concept presented by Sotelo et al. [8]. The T-LESS approach for distal ureter management can be applied either for single-port LNU or standard mul- 
tiport laparoscopy. The availability of a relatively easy closure of the bladder wall defect, made through a small skin incision, substantially minimizes the risk of complications related to urine extravasation, and also significantly limits the invasiveness of the LNU, which is usually a very challenging procedure.

Laparoscopic nephroureterectomy with en-bloc distal ureter and bladder cuff excision, using the T-LESS approach, is an efficient and safe procedure. Advantages of the method are a well-visualized dissection of the distal ureter, closing the defect of the bladder fundus, the use of standard laparoscopic instruments, and a good cosmesis. Nevertheless, further multicenter experience and observations are needed to validate the procedure.

\section{References}

1. Clayman RV, Kavoussi LR, Figenshau RS, et al. Laparoscopic nephroureterectomy: initial clinical case report. J Laparoendoscopic Surg 1991; 1: 343-9.

2. Keeley FX Jr, Tolley DA. Laparoscopic nephroureterectomy: making management of upper-tract transitional-cell carcinoma entirely minimally invasive. J Endourol 1998; 12: 139-41.

3. Machado MT, Pinto MA, Juliano RV, et al. Preliminary experience with ureteral intussusception in exclusive retroperitoneoscopic nephrectomy: a simple and safe option for the resection of the distal ureter and bladder cuff. Arch Esp Urol 2002; 55: 582-6.

4. Shalhav AL, Elbahnasy AM, McDougall EM, et al. Laparoscopic nephroureterectomy for upper tract transitional-cell cancer: technical aspects. J Endourol 1998; 12: 345-53.

5. Gill IS, Soble JJ, Miller SD, et al. A novel technique for management of the en bloc bladder cuff and distal ureter during laparoscopic nephroureterectomy. J Urol 1999; 161: 430-4.

6. Cheng CW, Ng CF, Mak SK, et al. Pneumovesicum method in en-bloc laparoscopic nephroureterectomy with bladder cuff resection for upper-tract urothelial cancer. J Endourol 2007; 21 359-62.

7. Guzzo TJ, Schaeffer EM, Allaf ME. Laparoscopic radical nephroureterectomy with en-bloc distal ureteral and bladder cuff excision using a single position pneumovesicum method. Urology 2008; 72: 850-2.

8. Sotelo R, Ramirez D, Carmona O, et al. A novel technique for distal ureterectomy and bladder cuff excision. Actas Urol Esp 2011; 35: 168-74.

9. Krogh J, Kvist E, Rye B. Transitional cell carcinoma of the upper urinary tract: prognostic variables and postoperative recurrences. Br J Urol 1991; 67: 32-6.

10. Hall M, Womack S, Sagalowsky A. Prognostic factors, recurrence and survival in transitional cell carcinoma of the upper urinary tract: a 30-year experience in 252 patients. Urology 1998; 52: 594-601.

11. Tsujihata M, Nonomura N, Tsujimura A, et al. Laparoscopic nephroureterectomy for upper tract transitional cell carcinoma: comparison of laparoscopic and open surgery. Eur Urol 2006; 49: $332-6$

12. Gill IS, Sung GT, Hobart MG, et al. Laparoscopic radical nephroureterectomy for upper tract transitional cell carcinoma: the Cleveland Clinic experience. J Urol 2000; 164: 1513-22.

13. Shalhav AL, Dunn MD, Portis AJ, et al. Laparoscopic nephroureterectomy for upper tract transitional cell cancer: the Washington University experience. J Urol 2000; 163: 1100-4.

14. Matin SF, Gill IS. Recurrence and survival following laparoscopic radical nephroureterectomy with various forms of bladder cuff control. J Urol 2005; 173: 395-400.

15. Tsivian A, Benjamin S, Sidi AA. A sealed laparoscopic nephroureterectomy: a new technique. Eur Urol 2007; 52: 1015-9.

16. Mak SK, Ng CF, Chan ESY, et al. Pneumovesicum approach to en-bloc laparoscopic nephroureterectomy with bladder cuff excision for upper tract urothelial cancer: midterm oncological results. J Endourol 2011; 25: 611-4.

17. Hattori R, Yoshino Y, Komatsu T, et al. Pure laparoscopic complete excision of distal ureter with a bladder cuff for upper tract urothelial carcinoma. World J Urol 2009; 27: 253-8.

18. Ahlawat RK, Gautam G. Suprapubic transvesical single-port technique for control of lower end of ureter during laparoscopic nephroureterectomy for upper tract transitional cell carcinoma. Ind J Urol 2011; 27: 190-5.

19. Zou X, Zhang G, Wang X, et al. A one-port pneumovesicum method in en bloc laparoscopic nephroureterectomy with bladder cuff resection is feasible and safe for upper tract transitional cell carcinoma. BJU Int 2011; 108: 1497-500.

20. Wong C, Leveilee RJ. Hand-assisted laparoscopic nephroureterectomy with cystoscopic en bloc excision of the distal ureter and bladder cuff. J Endourol 2002; 16: 329-33.

21. Desai MM, Fareed K, Berger AK, et al. Single-port transvesical enucleation of the prostate: a clinical report of 34 cases. BJU Int 2010; 105: 1296-300.

22. Roslan M, Markuszewski M, Kłącz J, et al. Laparoendoscopic single-port transvesical diverticulectomy: preliminary clinical experience. J Endourol 2012; 26: 975-9.

23. Ingber M, Stein R, Rackley R, et al. Single-port transvesical excision of foreign body in the bladder. Urology 2009; 74: 1347-50.

24. Roslan M, Markuszewski M, Kłącz J, et al. Laparoendoscopic single-site transvesical ureteroneocystostomy for vesicoureteral reflux in an adult: a one-year follow-up. Urology 2012; 80: 719-23.

25. Szopiński TR, Sudoł-Szopińska I, Furmanek MI, et al. Degeneration of the symphysis pubis presenting as a submucosal urinary bladder tumour. Videosurgery Miniinv 2012; 7: 55-8.

Received: 24.06.2013, accepted: 24.09.2013. 